\title{
Determination of the real poles of the Igusa zeta function for curves
}

\author{
Denis Ibadula* Dirk Segers ${ }^{\dagger}$
}

April 27, 2011

\begin{abstract}
The numerical data of an embedded resolution determine the candidate poles of Igusa's $p$-adic zeta function. We determine in complete generality which real candidate poles are actual poles in the curve case.
\end{abstract}

\section{Introduction}

Several mathematicians have already obtained partial results about the determination of the poles of Igusa's p-adic zeta function for curves. In this paper, we will determine the real poles for an arbitrary polynomial $f$ in two variables which is defined over a $p$-adic field. People are interested in the poles of Igusa's $p$-adic zeta function $Z_{f}(s)$ because they determine the asymptotic behaviour of the number of solutions of polynomial congruences and because they are the subject of the monodromy conjecture (see for example [De2]).

Historically, one considered first only curves which are absolutely analytically irreducible. Partial results were obtained by Igusa [Ig1] and Strauss [St]. Meuser [Me] determined the real poles, but she did not consider the candidate pole -1 . In 1985 Igusa [Ig2] solved that problem completely. He proved that the candidate poles associated to the strict transform of $f$ are poles when the domain of integration is small enough. Moreover, another candidate pole of the minimal embedded resolution of $f$ is a pole if and only if it is associated to an exceptional curve which is intersected by three other irreducible components of the pull-back of $f$. We have incorporated a generalization of this result (Proposition 2).

\footnotetext{
*This paper is supported by the Sectorial Operational Programme Human Resources Development (SOP HRD), financed from the European Social Fund and by the Romanian Government under the contract number SOP HRD/89/1.5/S/62988.

${ }^{\dagger}$ Postdoctoral Fellow of the Fund for Scientific Research - Flanders (Belgium). 2010 Mathematics Subject Classification. 11D79 11S80 14B05 14E15 Key words. Igusa's $p$-adic zeta function.
} 
In the general case, Loeser [Lo] obtained that an exceptional curve $E_{i}$ does not contribute to the poles of $Z_{f}(s)$ if $E_{i}$ is intersected one or two times by other components of the pull-back of $f$ and if there are no other intersection points over an algebraic closure. This was first proved by Strauss in the absolutely analytically irreducible case, where the last condition is automatically satisfied.

The next paper we want to mention is [Ve1] of Veys. He considers a polynomial $f$ in two variables over a number field $F$ and takes the minimal embedded resolution of $f$ over an algebraic closure of $F$. This setup allowed him to use a formula [De1] of Denef for $Z_{f}(s)$, which is valid for almost all $p$-adic completions of $F$. He supposes that all intersection points of irreducible components of the pull-back of $f$ are defined over $F$. Under this condition, he proves the converse of the result of Loeser for real candidate poles and for almost all $p$-adic completions of $F$. Moreover, he deals with the problem of a possible cancellation of several contributions to the same real candidate pole.

In the proofs of the mentioned vanishing and non-vanishing results, one needed certain relations between the various numerical data of the embedded resolution. They were systematically derived in $[\mathrm{St}],[\mathrm{Me}]$ and [Ig2] for absolutely analytically irreducible curves and finally, Loeser [Lo] obtained the necessary relations in the general case. Igusa [Ig2] and Loeser [Lo] used a formula of Langlands [La] to calculate the contribution of an exceptional curve to the residue of $Z_{f}(s)$ at a candidate pole of candidate order one. We will use a slight variant of this formula which was obtained in [Se1]. Given an embedded resolution written as a composition of blowing-ups, the second author explained there how to calculate this contribution to the residue at the stage where the exceptional curve is created. In Proposition 1, we determine when this contribution is zero and when not. For this, we need new ideas. It is not at all a straightforward generalization of what was already known. Finally in Section 4, we will prove that contributions to the same candidate pole will not cancel out. For this, we use that the dual embedded resolution graph is an ordered tree. This was obtained in [Ve2] when the base field is algebraically closed.

Acknowledgements. The first author is grateful to Willem Veys for inviting her to an academic visit at the University of Leuven in 2009. During this visit the work to this paper was started. We also want to thank Bart Bories and Willem Veys for the conversations that we had with them concerning this paper and for their remarks.

\section{Definitions and our tools}

Let $K$ be a $p$-adic field, i.e., an extension of $\mathbb{Q}_{p}$ of finite degree. Let $R$ be the valuation ring of $K, P$ the maximal ideal of $R$ and $q$ the cardinality of the residue field $R / P$. For $z \in K$, let ord $z \in \mathbb{Z} \cup\{+\infty\}$ denote the valuation of $z$ 
and $|z|=q^{- \text {ord } z}$ the absolute value of $z$.

Let $f\left(x_{1}, x_{2}\right) \in K\left[x_{1}, x_{2}\right]$ be a polynomial in two variables over $K$ and put $x=\left(x_{1}, x_{2}\right)$. Let $X$ be an open and compact subset of $K^{2}$. Igusa's $p$-adic zeta function of $f$ is defined by

$$
Z_{f}(s)=\int_{X}|f(x)|^{s}|d x|
$$

for $s \in \mathbb{C}, \operatorname{Re}(s)>0$, where $|d x|$ denotes the Haar measure on $K^{2}$, normalised so that $R^{2}$ has measure 1 . Igusa proved that $Z_{f}(s)$ is a rational function of $q^{-s}$ by calculating the integral on an embedded resolution of $f$. Therefore, it extends to a meromorphic function $Z_{f}(s)$ on $\mathbb{C}$ which is also called Igusa's $p$-adic zeta function of $f$.

Let $g: Y \rightarrow X$ be an embedded resolution of $f$. Here, $Y$ is a $K$-analytic manifold. The meaning of embedded resolution in our context is explained in [Ig3, Section 3.2]. Write $g=g_{1} \circ \cdots \circ g_{t}: Y=Y_{t} \rightarrow X=Y_{0}$ as a composition of blowing-ups $g_{i}: Y_{i} \rightarrow Y_{i-1}, i \in T_{e}:=\{1, \ldots, t\}$. The exceptional curve of $g_{i}$ and also the strict transforms of this curve are denoted by $E_{i}$. The closed submanifolds of $Y$ of codimension one which are the zero locus of the strict transform of an irreducible factor of $f$ in $K[x, y]$ are denoted by $E_{j}, j \in T_{s}$. The corresponding transforms in $Y_{i}, i \in\{0, \ldots, t-1\}$, are denoted in the same way. Note that we had to be careful with the notion of irreducible, because $X$ is totally disconnected as a topological space. Put $T=T_{e} \cup T_{s}$. For $i \in T$, let $N_{i}$ and $\nu_{i}-1$ be the multiplicities of respectively $f \circ g$ and $g^{*} d x$ along $E_{i}$. The $\left(N_{i}, \nu_{i}\right)$ are called the numerical data of $E_{i}$.

Let us recall Igusa's proof of the rationality of $Z_{f}(s)$. As we already said, we calculate the defining integral on $Y$ :

$$
Z_{f}(s)=\int_{X}|f(x)|^{s}|d x|=\int_{Y}|f \circ g|^{s}\left|g^{*} d x\right| .
$$

Let $b$ be an arbitrary point of $Y$. There are three cases. In the first case, there are two varieties $E_{i}$ and $E_{j}$, with $i, j \in T$, that pass through $b$. We take a neighborhood $V$ of $b$ and analytic coordinates $\left(y_{1}, y_{2}\right)$ on $V$ such that $y_{1}$ is an equation of $E_{i}, y_{2}$ is an equation of $E_{j}$,

$$
f \circ g=\varepsilon y_{1}^{N_{i}} y_{2}^{N_{j}} \quad \text { and } \quad g^{*} d x=\eta y_{1}^{\nu_{i}-1} y_{2}^{\nu_{j}-1} d y
$$

on $V$ for non-vanishing $K$-analytic functions $\varepsilon$ and $\eta$ on $V$. We may suppose that $y(V)=P^{k_{1}} \times P^{k_{2}}$, with $k_{1}, k_{2} \in \mathbb{Z}_{\geq 0}$, and that $|\varepsilon|$ and $|\eta|$ are constant on $V$. We get

$$
\begin{aligned}
\int_{V}|f \circ g|^{s}\left|g^{*} d x\right| & =\int_{P^{k_{1}} \times P^{k_{2}}}|\varepsilon|^{s}|\eta|\left|y_{1}\right|^{N_{i} s+\nu_{i}-1}\left|y_{2}\right|^{N_{j} s+\nu_{j}-1}|d y| \\
& =|\varepsilon|^{s}|\eta|\left(\frac{q-1}{q}\right)^{2} \frac{q^{-k_{1}\left(N_{i} s+\nu_{i}\right)}}{1-q^{-\left(N_{i} s+\nu_{i}\right)}} \frac{q^{-k_{2}\left(N_{j} s+\nu_{j}\right)}}{1-q^{-\left(N_{j} s+\nu_{j}\right)}}
\end{aligned}
$$


Note that this is a rational function of $q^{-s}$. In the second case, there is one variety $E_{i}, i \in T$, that passes through $b$. We take a neighborhood $V$ of $b$ and analytic coordinates $\left(y_{1}, y_{2}\right)$ on $V$ such that $y_{1}$ is an equation of $E_{i}$,

$$
f \circ g=\varepsilon y_{1}^{N_{i}} \quad \text { and } \quad g^{*} d x=\eta y_{1}^{\nu_{i}-1} d y
$$

on $V$ for non-vanishing $K$-analytic functions $\varepsilon$ and $\eta$ on $V$. We may suppose that $y(V)=P^{k_{1}} \times P^{k_{2}}$, with $k_{1}, k_{2} \in \mathbb{Z}_{\geq 0}$, and that $|\varepsilon|$ and $|\eta|$ are constant on $V$. We get

$$
\begin{aligned}
\int_{V}|f \circ g|^{s}\left|g^{*} d x\right| & =\int_{P^{k_{1}} \times P^{k_{2}}}|\varepsilon|^{s}|\eta|\left|y_{1}\right|^{N_{i} s+\nu_{i}-1}|d y| \\
& =|\varepsilon|^{s}|\eta| q^{-k_{2}} \frac{q-1}{q} \frac{q^{-k_{1}\left(N_{i} s+\nu_{i}\right)}}{1-q^{-\left(N_{i} s+\nu_{i}\right)}} .
\end{aligned}
$$

In the third case, there is no variety $E_{i}, i \in T$, that passes through $b$. We take a neighborhood $V$ of $b$ and analytic coordinates $\left(y_{1}, y_{2}\right)$ on $V$ such that $f \circ g=\varepsilon$ and $g^{*} d x=\eta d y$ on $V$ for non-vanishing $K$-analytic functions $\varepsilon$ and $\eta$ on $V$. We may suppose that $y(V)=P^{k_{1}} \times P^{k_{2}}$, with $k_{1}, k_{2} \in \mathbb{Z}_{\geq 0}$, and that $|\varepsilon|$ and $|\eta|$ are constant on $V$. We get

$$
\int_{V}|f \circ g|^{s}\left|g^{*} d x\right|=|\varepsilon|^{s}|\eta| q^{-k_{1}-k_{2}}
$$

It follows now that $Z_{f}(s)$ is a rational function of $q^{-s}$ because we can partition $Y$ into sets $V$ of the above form.

We obtain also from this calculation that every pole of $Z_{f}(s)$ is of the form

$$
-\frac{\nu_{i}}{N_{i}}+\frac{2 k \pi \sqrt{-1}}{N_{i} \log q}
$$

with $k \in \mathbb{Z}$ and $i \in T$. These values are called the candidate poles of $Z_{f}(s)$. If $i \in T$ is fixed, the values $-\nu_{i} / N_{i}+(2 k \pi \sqrt{-1}) /\left(N_{i} \log q\right), k \in \mathbb{Z}$, are called the candidate poles of $Z_{f}(s)$ associated to $E_{i}$. Because the poles of $1 /\left(1-q^{-N_{i} s-\nu_{i}}\right)$ have order one, we define the expected order of a candidate pole $s_{0}$ as the highest number of $E_{i}$ 's with candidate pole $s_{0}$ and with non-empty intersection. The order of $s_{0}$ is of course less than or equal to its expected order and a candidate pole $s_{0}$ of expected order one is a pole if and only if the residue of $Z_{f}(s)$ at $s_{0}$ is different from 0 .

Let us explain the formula for the residue that we will use. Let $s_{0}$ be a candidate pole of $E_{i}, i \in T$, and suppose that $s_{0}$ is not a candidate pole of any $E_{j}$, with $j \in T$ and $j \neq i$, which intersects $E_{i}$ in $Y$. Let $U$ be an open and compact subset of $E_{i}$. The contribution of $U$ to the residue of $Z_{f}(s)$ at $s_{0}$ is by definition the contribution to the residue of $Z_{f}(s)$ at $s_{0}$ of an open and compact subset $V$ of $Y$ which satisfies $V \cap E_{i}=U$ and which is disjoint from every other $E_{j}$ 
with candidate pole $s_{0}$. Suppose that $U$ already exists in $Y_{r}$ and if $i \in T_{s}$ we also suppose that it is non-singular in $Y_{r}$. Suppose that $W$ is an open and compact subset of $Y_{r}$ for which $W \cap E_{i}=U$ and that $\left(z_{1}, z_{2}\right)$ are analytic coordinates on $W$ such that $z_{1}=0$ is an equation of $U$ on $W$. Write

$$
f \circ g_{1} \circ \cdots \circ g_{r}=\gamma z_{1}^{N_{i}} \quad \text { and } \quad\left(g_{1} \circ \cdots \circ g_{r}\right)^{*} d x=\delta z_{1}^{\nu_{i}-1} d y
$$

on $W$, for $K$-analytic functions $\gamma$ and $\delta$ on $W$. Then, the contribution of $U$ to the residue of $Z_{f}(s)$ at $s_{0}$ is equal to

$$
\frac{q-1}{q N_{i} \log q}\left[\int_{U}|\gamma|^{s}|\delta|\left|d z_{2}\right|\right]_{s=s_{0}}^{\mathrm{mc}}
$$

where $[\cdot]_{s=s_{0}}^{\mathrm{mc}}$ denotes the evaluation in $s=s_{0}$ of the meromorphic continuation of the function between the brackets. This formula was obtained by Langlands [La] in the case $r=t$ and in general by the second author in [Se1, Section 2.6].

We explain now the relations that we will need. Fix $r \in T_{e}$. The exceptional curve $E_{r}$ is obtained by blowing-up at a point $P \in Y_{r-1}$. Let $y=\left(y_{1}, y_{2}\right)$ be local coordinates on $Y_{r-1}$ centered at $P$. Write in these local coordinates

$f \circ g_{1} \circ \cdots \circ g_{r-1}=d\left(\prod_{i \in S}\left(a_{i 2} y_{1}-a_{i 1} y_{2}\right)^{M_{i}}\right)\left(\prod_{i \in S^{\prime}} h_{i}^{M_{i}}\left(y_{1}, y_{2}\right)\right)+$ terms of higher degree,

where all factors $a_{i 2} y_{1}-a_{i 1} y_{2}$ and $h_{i}$ are essentially different (i.e. no factor is equal to another multiplied by an element of $K^{\times}$) polynomials over $K$, where the $h_{i}$ are irreducible homogeneous polynomials of degree at least two, where $M_{i} \geq 1$ for every $i \in S \cup S^{\prime}$ and where $d \in K^{\times}$. Write also

$$
\left(g_{1} \circ \cdots \circ g_{r-1}\right)^{*} d x=\left(e \prod_{i \in S}\left(a_{i 2} y_{1}-a_{i 1} y_{2}\right)^{\mu_{i}-1}+\text { terms of higher degree }\right) d y,
$$

where $\mu_{i} \geq 1$ for every $i \in S$ and $e \in K^{\times}$. Let $s_{0}=-\nu_{r} / N_{r}+(2 k \pi \sqrt{-1}) /\left(N_{r} \log q\right)$ be an arbitrary candidate pole of $Z_{f}(s)$ associated to $E_{r}$. We advise the reader to specialize everything what follows in this section to the case $k=0$. Put $\alpha_{i}:=\mu_{i}+s_{0} M_{i}$ for every $i \in S$. Because

$$
N_{r}=\sum_{i \in S} M_{i}+\sum_{i \in S^{\prime}}\left(\operatorname{deg} h_{i}\right) M_{i} \quad \text { and } \quad \nu_{r}=\sum_{i \in S}\left(\mu_{i}-1\right)+2,
$$

it is straightforward to check that

$$
\sum_{i \in S}\left(\alpha_{i}-1\right)+\sum_{i \in S^{\prime}} s_{0}\left(\operatorname{deg} h_{i}\right) M_{i}=-2+\frac{2 k \pi \sqrt{-1}}{\log q} .
$$


We now give another description of the $\alpha_{i}$. Let $F_{i}$ be the point on $E_{r}$ which has coordinates $\left(a_{i 1}: a_{i 2}\right)$ with respect to the homogenous coordinates $\left(y_{1}: y_{2}\right)$ on $E_{r} \subset Y_{r}$. Let $j$ be the unique element of $T \backslash\{r\}$ such that $E_{j}$ passes through $F_{i}$ in $Y$. Let $\rho$ be the number of blowing-ups among $g_{r}, \ldots, g_{t}$ which are centered at $F_{i}$. Then, the announced description is $\alpha_{i}=\nu_{j}+s_{0} N_{j}-(2 \rho k \pi \sqrt{-1}) /(\log q)$. The second author proved this in [Se1, Section 2.7] in the case $k=0$, and the general case is treated in a similar way. It follows that $\operatorname{Re}\left(\alpha_{i}\right)<0$ if and only if $-\nu_{r} / N_{r}<-\nu_{j} / N_{j}$. One checks also easily that

$s_{0}$ is a candidate pole of $E_{j} \Longleftrightarrow \nu_{j}+s_{0} N_{j}$ is a multiple of $2 \pi \sqrt{-1} /(\log q)$

$\Longleftrightarrow \alpha_{i}$ is a multiple of $2 \pi \sqrt{-1} /(\log q)$.

It is proved in [Lo, Proposition II.3.1] that $\operatorname{Re}\left(\alpha_{i}\right)<1$. Together with (2), this implies that $\operatorname{Re}\left(\alpha_{i}\right) \geq-1$ and that there is at most one $i \in S$ with $\operatorname{Re}\left(\alpha_{i}\right)<0$.

\section{Contribution of one exceptional curve}

Setting of Proposition 1 and 2. Let $f \in K\left[x_{1}, x_{2}\right]$ and let $X$ be an open and compact subset of $K^{2}$. Let $g: Y \rightarrow X$ be an embedded resolution of $f$. Write $g=g_{1} \circ \cdots \circ g_{t}: Y=Y_{t} \rightarrow X=Y_{0}$ as a composition of blowing-ups $g_{i}: Y_{i} \rightarrow Y_{i-1}$, $i \in T_{e}:=\{1, \ldots, t\}$. The exceptional curve of $g_{i}$ and also the strict transforms of this curve are denoted by $E_{i}$. Let $r \in T_{e}$. The exceptional curve $E_{r}$ is obtained by blowing-up at a point $P \in Y_{r-1}$. Let $\left(y_{1}, y_{2}\right)$ be local coordinates on $Y_{r-1}$ centered at $P$. Write in these local coordinates

$f \circ g_{1} \circ \cdots \circ g_{r-1}=d\left(\prod_{i \in S}\left(a_{i 2} y_{1}-a_{i 1} y_{2}\right)^{M_{i}}\right)\left(\prod_{i \in S^{\prime}} h_{i}^{M_{i}}\left(y_{1}, y_{2}\right)\right)+$ terms of higher degree,

where all factors $a_{i 2} y_{1}-a_{i 1} y_{2}$ and $h_{i}$ are essentially different polynomials over $K$, where the $h_{i}$ are irreducible homogeneous polynomials of degree at least two, where $M_{i} \geq 1$ for every $i \in S \cup S^{\prime}$ and where $d \in K^{\times}$. Write also

$$
\left(g_{1} \circ \cdots \circ g_{r-1}\right)^{*} d x=\left(e \prod_{i \in S}\left(a_{i 2} y_{1}-a_{i 1} y_{2}\right)^{\mu_{i}-1}+\text { terms of higher degree }\right) d y,
$$

where $\mu_{i} \geq 1$ for every $i \in S$ and $e \in K^{\times}$.

Proposition 1. Let $s_{0}:=-\nu_{r} / N_{r}$ be the real candidate pole of $Z_{f}(s)$ associated to $E_{r}$. Suppose that $\alpha_{i}:=\mu_{i}+s_{0} M_{i} \neq 0$ for every $i \in S$. Let $\mathcal{R}$ be the contribution of $E_{r}$ to the residue of $Z_{f}(s)$ at $s_{0}$. Then, $\mathcal{R} \neq 0$ if and only if $|S| \geq 3$ or $\left|S^{\prime}\right| \geq 1$. Moreover, if $\mathcal{R} \neq 0$, then

1. $\mathcal{R}>0$ if and only if $\alpha_{i}>0$ for every $i \in S$ and

2. $\mathcal{R}<0$ if and only if $\alpha_{i}<0$ for some (and thus exactly one) $i \in S$. 
Proof. If the number of elements of $S$ is one or two and $S^{\prime}$ is empty, then it is well known that $\mathcal{R}=0$. We mentioned already in the introduction that Loeser [Lo] proved this by using the formula of Langlands, and the second author proved this again [Se1, Section 3.1] as an illustration of his variant of this formula.

Suppose from now on that $|S| \geq 3$ or $\left|S^{\prime}\right| \geq 1$. We consider first the case in which there exists one element $l \in S$ satisfying $\alpha_{l}<0$. Denote $Q:=S \backslash\{l\}$ and $Q^{\prime}=S^{\prime}$. By applying an affine coordinate transformation, we may assume that

$f \circ g_{1} \circ \cdots \circ g_{r-1}=d\left(y_{2}^{M_{l}} \prod_{i \in Q}\left(y_{1}-a_{i} y_{2}\right)^{M_{i}}\right)\left(\prod_{i \in Q^{\prime}} h_{i}^{M_{i}}\left(y_{1}, y_{2}\right)\right)+$ terms of higher degree,

and

$$
\left(g_{1} \circ \cdots \circ g_{r-1}\right)^{*} d x=\left(e y_{2}^{\mu_{l}-1} \prod_{i \in Q}\left(y_{1}-a_{i} y_{2}\right)^{\mu_{i}-1}+\text { terms of higher degree }\right) d y,
$$

where the $a_{i}, i \in Q$, are different elements of $R$, where $h_{i}, i \in Q^{\prime}$, are different irreducible homogeneous polynomials over $R$ of degree $d_{i} \geq 2$ with coefficient of $y_{1}^{d_{i}}$ equal to 1 , where $M_{i} \geq 1$ for every $i \in Q \cup Q^{\prime}$ and where $d, e \in K^{\times}$. We have that $\mathcal{R}$ is the sum of two contributions, which we calculate on two different charts by using formula (1). For the first contribution, we look at the coordinates $\left(z_{1}, z_{2}\right)$ on $Y_{r}$ for which $g_{r}\left(z_{1}, z_{2}\right)=\left(z_{1}, z_{1} z_{2}\right)$, and obtain $\kappa:=(q-1) /\left(q N_{r} \log q\right)$ times

$$
\begin{aligned}
{\left[|d|^{s}|e| \int_{P}\left|z_{2}\right|^{M_{l} s+\mu_{l}-1} \prod_{i \in Q}\right.} & \left.\left(\left|1-a_{i} z_{2}\right|^{M_{i} s+\mu_{i}-1}\right)\left(\prod_{i \in Q^{\prime}}\left|h_{i}\left(1, z_{2}\right)\right|^{M_{i} s}\right)\left|d z_{2}\right|\right]_{s=s_{0}}^{\mathrm{mc}} \\
& =|d|^{s_{0}}|e|\left[\int_{P}\left|z_{2}\right|^{M_{l} s+\mu_{l}-1}\left|d z_{2}\right|\right]_{s=s_{0}}^{\mathrm{mc}} \\
& =|d|^{s_{0}}|e| \frac{q-1}{q} \frac{1}{q^{\alpha_{l}}-1} .
\end{aligned}
$$

For the second contribution, we look at the coordinates $\left(z_{1}^{\prime}, z_{2}^{\prime}\right)$ on $Y_{r}$ for which $g_{r}\left(z_{1}^{\prime}, z_{2}^{\prime}\right)=\left(z_{1}^{\prime} z_{2}^{\prime}, z_{2}^{\prime}\right)$, and obtain $\kappa$ times

$$
\left[|d|^{s}|e| \int_{R} \prod_{i \in Q}\left(\left|z_{1}^{\prime}-a_{i}\right|^{M_{i} s+\mu_{i}-1}\right)\left(\prod_{i \in Q^{\prime}}\left|h_{i}\left(z_{1}^{\prime}, 1\right)\right|^{M_{i} s}\right)\left|d z_{1}^{\prime}\right|\right]_{s=s_{0}}^{\mathrm{mc}},
$$

which is according to Lemma 3 less than $\kappa$ times

$$
|d|^{s_{0}}|e|\left[\int_{R}\left|z_{1}^{\prime}-a\right|^{M s+\mu-1}\left|d z_{1}^{\prime}\right|\right]_{s=s_{0}}^{\mathrm{mc}}=|d|^{s_{0}}|e| \frac{q-1}{q} \frac{1}{1-q^{-\left(M s_{0}+\mu\right)}},
$$


where $a \in R, M:=\left(\sum_{i \in Q} M_{i}\right)+\left(\sum_{i \in Q^{\prime}} d_{i} M_{i}\right)$ and $\mu:=\left(\sum_{i \in Q}\left(\mu_{i}-1\right)\right)+1$. By using that $\alpha_{l}+\left(M s_{0}+\mu\right)=0$, we obtain

$$
\frac{1}{q^{\alpha_{l}}-1}+\frac{1}{1-q^{-\left(M s_{0}+\mu\right)}}=0
$$

and this implies that $\mathcal{R}<0$.

Consider now the case in which $\alpha_{i}>0$ for every $i \in S$. This case is much easier. After calculating $\mathcal{R}$ analogously as in the previous case by using formula (1), you see that $\mathcal{R}$ is a sum of positive numbers and thus positive. You have to use that $|h|$ is a locally constant function for an irreducible polynomial $h$ over $K$ in one variable of degree at least 2.

We still have to prove Lemma 3. First, we prove Lemma 2, which is a special case of Lemma 3. In the proof of Lemma 2, we need Lemma 1.

Lemma 1. Let $h \in R[x]$ be an irreducible monic polynomial of degree $d \geq 2$ in one variable. Then, there exists a unique $r \in \mathbb{N}$ and an element $b$ in $R$ such that

$$
|h(x)|=\left|(x-b)^{d}\right| \quad \text { if } x \in R \text { and } x \not \equiv b \bmod P^{r}
$$

and

$$
q^{-d r} \leq|h(x)|<q^{-d(r-1)} \quad \text { if } x \in R \text { and } x \equiv b \bmod P^{r} .
$$

Moreover, $b$ is only determined modulo $P^{r}$ and $|h(x)|$ is constant on $b+P^{r}$.

Proof. Let $\beta_{1}, \ldots, \beta_{d}$ be the roots of $h$ in an algebraic closure of $K$. Let $L:=$ $K\left(\beta_{1}, \ldots, \beta_{d}\right)$, let $R_{L}$ be the valuation ring of $L$ and denote the extension of the norm on $K$ to $L$ also by $|\cdot|$. Note that $\beta_{1}, \ldots, \beta_{d}$ are different because we work in characteristic zero and that they are in $R_{L}$ because $h$ is monic and $R_{L}$ is the integral closure of $R$ in $L$. Because

$$
\begin{aligned}
|h(x)| & =\left|\left(x-\beta_{1}\right)\left(x-\beta_{2}\right) \cdots\left(x-\beta_{d}\right)\right| \\
& =\left|x-\beta_{1}\right|\left|x-\beta_{2}\right| \cdots\left|x-\beta_{d}\right|,
\end{aligned}
$$

we look at $\left|x-\beta_{i}\right|$.

Take $i \in\{1, \ldots, d\}$. Let $r_{i}$ be the largest natural number for which there exists an element $b_{i} \in R$ satisfying $\left|b_{i}-\beta_{i}\right|<q^{-\left(r_{i}-1\right)}$. Note that this largest natural number exists because $\beta_{i} \notin R$. Note also that $b_{i}$ is only determined modulo $P^{r_{i}}$. One checks now that

$$
\left|x-\beta_{i}\right|=\left|x-b_{i}\right| \quad \text { if } x \in R \text { and } x \not \equiv b_{i} \bmod P^{r_{i}}
$$

and

$$
q^{-r_{i}} \leq\left|x-\beta_{i}\right|<q^{-\left(r_{i}-1\right)} \quad \text { if } x \in R \text { and } x \equiv b_{i} \bmod P^{r_{i}}
$$


Moreover, $\left|x-\beta_{i}\right|$ is constant on $b_{i}+P^{r_{i}}$.

Finally, we show that $r_{1}=r_{2}=\cdots=r_{d}$ and we will denote this by $r$. We also show that the $b_{i}, i \in\{1, \ldots, d\}$, are the same modulo $P^{r}$, and because the $b_{i}$ are only determined modulo $P^{r}$, we can take $b_{1}=b_{2}=\cdots=b_{d}$ and we will denote this by $b$. Then the lemma obviously follows.

Take $i, j \in\{1, \ldots, d\}$ and suppose that $r_{i} \geq r_{j}$. Because $\beta_{i}$ and $\beta_{j}$ are conjugate and because $b_{i} \in R$, we get that also $b_{i}-\beta_{i}$ and $b_{i}-\beta_{j}$ are conjugate. Because conjugate numbers have the same norm, we get $\left|b_{i}-\beta_{j}\right|=\left|b_{i}-\beta_{i}\right|<$ $q^{-\left(r_{i}-1\right)}$. This implies that $r_{i} \leq r_{j}$ and thus $r_{i}=r_{j}$ and that $b_{i} \equiv b_{j} \bmod P^{r_{i}}$.

Lemma 2. Let $s_{0}$ be a negative rational number. Let $a_{1}, \ldots, a_{k}$ be different elements of $R$ and suppose that we have for every $i \in\{1, \ldots, k\}$ integers $M_{i} \geq 1$ and $\mu_{i} \geq 1$ satisfying $\alpha_{i}:=\mu_{i}+s_{0} M_{i}<1$. Let $h_{k+1}, \ldots, h_{l} \in R[x]$ be different irreducible monic polynomials in one variable of degree at least two. Denote the degree of $h_{i}$ by $d_{i}$ and suppose that we have for every $i \in\{k+1, \ldots, l\}$ an integer $M_{i} \geq 1$. Let $r_{i}$ be the natural number which we associated to $h_{i}$ in the previous lemma, and $b_{i}$ a corresponding element of $R$ which is determined modulo $P^{r_{i}}$. Suppose that $r_{k+1}=\cdots=r_{l}$ and denote this number by $r$. Suppose also that $a_{1} \equiv \cdots \equiv a_{k} \equiv b_{k+1} \equiv \cdots \equiv b_{l} \bmod P^{r}$ and that $a_{i} \not \equiv a_{j} \bmod P^{r+1}$ for $i \neq j$. Take now $a \in R$ such that $a \equiv a_{1} \bmod P^{r}$ and put $M:=M_{1}+\cdots+M_{k}+$ $d_{k+1} M_{k+1}+\cdots+d_{l} M_{l}, \mu:=\left(\mu_{1}-1\right)+\cdots+\left(\mu_{k}-1\right)+1$ and $\alpha:=\mu+s_{0} M$. Suppose that $0<\alpha$ and that $k \geq 2$ or $l \geq k+1$. Then

$$
\begin{gathered}
{\left[\int_{a+P^{r}}\left|x-a_{1}\right|^{M_{1} s+\mu_{1}-1} \cdots\left|x-a_{k}\right|^{M_{k} s+\mu_{k}-1}\left|h_{k+1}(x)\right|^{M_{k+1} s} \ldots\left|h_{l}(x)\right|^{M_{l} s}|d x|\right]_{s=s_{0}}^{\mathrm{mc}}} \\
<\left[\int_{a+P^{r}}|x-a|^{M s+\mu-1}|d x|\right]_{s=s_{0}}^{\mathrm{mc}} \cdot
\end{gathered}
$$

Moreover, the integrands are the same for every $x \in R \backslash\left(a+P^{r}\right)$.

Remark. (1) The conditions $a_{i} \equiv a_{j} \bmod P^{r}$ and $a_{i} \not \equiv a_{j} \bmod P^{r+1}$ for $i, j \in$ $\{1, \ldots, k\}$ with $i \neq j$ imply that $k \leq q$.

(2) We have that

$$
\alpha-1=\sum_{i=1}^{k}\left(\alpha_{i}-1\right)+\sum_{i=k+1}^{l} s_{0} d_{i} M_{i} .
$$

Consequently, the condition $\alpha_{i}<1$ for every $i \in\{1, \ldots, k\}$ implies that $\alpha<\alpha_{i}$.

Proof. In the first step, we reduce to the case in which the polynomials $h_{i}$ do not occur. We have

$$
\left[\int_{a+P^{r}}|x-a|^{M s+\mu-1}|d x|\right]_{s=s_{0}}^{\mathrm{mc}}
$$




$$
\begin{aligned}
& =\frac{q-1}{q} \frac{q^{-r \alpha}}{1-q^{-\alpha}} \\
& =\frac{q-1}{q} \frac{q^{-r\left(\left(M_{1}+\cdots+M_{k}\right) s_{0}+\mu\right)}}{1-q^{-\alpha}} q^{-r\left(d_{k+1} M_{k+1}+\cdots+d_{l} M_{l}\right) s_{0}} \\
& \geq \frac{q-1}{q} \frac{q^{-r\left(\left(M_{1}+\cdots+M_{k}\right) s_{0}+\mu\right)}}{1-q^{-\left(\left(M_{1}+\cdots+M_{k}\right) s_{0}+\mu\right)}} q^{-r\left(d_{k+1} M_{k+1}+\cdots+d_{l} M_{l}\right) s_{0}} \\
& =q^{-r\left(d_{k+1} M_{k+1}+\cdots+d_{l} M_{l}\right) s_{0}}\left[\int_{a+P^{r}}|x-a|^{\left(M_{1}+\cdots+M_{k}\right) s+\mu-1}|d x|\right]_{s=s_{0}}^{\mathrm{mc}},
\end{aligned}
$$

with a strict inequality if $l \geq k+1$. Because $\left|h_{i}\right|$ is constant on $a+P^{r}$ with $q^{-d_{i} r} \leq\left|h_{i}(a)\right|, s_{0}<0$ and the second factor on the right hand side of the following inequality is positive (this follows from the calculation of this factor in the second part of the proof), we get

$$
\begin{aligned}
& {\left[\int_{a+P^{r}}\left|x-a_{1}\right|^{M_{1} s+\mu_{1}-1} \cdots\left|x-a_{k}\right|^{M_{k} s+\mu_{k}-1}\left|h_{k+1}(x)\right|^{M_{k+1} s} \ldots\left|h_{l}(x)\right|^{M_{l} s}|d x|\right]_{s=s_{0}}^{\mathrm{mc}}} \\
& \leq q^{-r\left(d_{k+1} M_{k+1}+\cdots+d_{l} M_{l}\right) s_{0}}\left[\int_{a+P^{r}}\left|x-a_{1}\right|^{M_{1} s+\mu_{1}-1} \cdots\left|x-a_{k}\right|^{M_{k} s+\mu_{k}-1}|d x|\right]_{s=s_{0}}^{\mathrm{mc}} .
\end{aligned}
$$

These two inequalities imply that it is enough to consider the case where the $h_{i}$ do not occur.

So in the second step, we prove that

$$
\left[\int_{a+P^{r}}|x-a|^{M s+\mu-1}|d x|\right]_{s=s_{0}}^{\mathrm{mc}}>\left[\int_{a+P^{r}}\left|x-a_{1}\right|^{M_{1} s+\mu_{1}-1} \cdots\left|x-a_{k}\right|^{M_{k} s+\mu_{k}-1}|d x|\right]_{s=s_{0}}^{\mathrm{mc}}
$$

if $k \geq 2$, where $M=M_{1}+\cdots+M_{k}$. We calculate both sides. We partition the domain of integration of the integral on the right hand side into the following $k+1$ sets: $a_{1}+P^{r+1}, \ldots, a_{k}+P^{r+1}$ and the set consisting of all other points of $a+P^{r}$. In this way, the above inequality becomes

$$
\begin{aligned}
\frac{q-1}{q} \frac{q^{-(r-1) \alpha}}{q^{\alpha}-1}> & \frac{q-1}{q} \frac{q^{-r \alpha_{1}}}{q^{\alpha_{1}}-1} q^{-r\left(\alpha_{2}-1\right)-r\left(\alpha_{3}-1\right)-\ldots-r\left(\alpha_{k}-1\right)}+\ldots \\
& +\frac{q-1}{q} \frac{q^{-r \alpha_{k}}}{q^{\alpha_{k}}-1} q^{-r\left(\alpha_{1}-1\right)-r\left(\alpha_{2}-1\right)-\ldots-r\left(\alpha_{k-1}-1\right)} \\
& +\frac{q-k}{q^{r+1}} q^{-r\left(\alpha_{1}-1\right)-r\left(\alpha_{2}-1\right)-\ldots-r\left(\alpha_{k}-1\right)} .
\end{aligned}
$$

By using the fact that $\alpha-1=\sum_{i=1}^{k}\left(\alpha_{i}-1\right)$, this is equivalent to

$$
(q-1) \frac{q^{\alpha}}{q^{\alpha}-1}>\frac{q-1}{q^{\alpha_{1}}-1}+\cdots+\frac{q-1}{q^{\alpha_{k}}-1}+q-k
$$


and thus also to

$$
\frac{1}{q^{\alpha}-1}+\frac{k-1}{q-1}>\frac{1}{q^{\alpha_{1}}-1}+\cdots+\frac{1}{q^{\alpha_{k}}-1}
$$

Consider the function

$$
h:] 0,1] \rightarrow \mathbb{R}: x \mapsto \frac{1}{q^{x}-1} .
$$

One checks easily that $h$ is convex, i.e. $h^{\prime \prime}(x)>0$ for every $\left.x \in\right] 0,1[$. Consider the linear function $g$, i.e. polynomial function of degree one, determined by $g(\alpha)=h(\alpha)=1 /\left(q^{\alpha}-1\right)$ and $g(1)=h(1)=1 /(q-1)$. Then

$$
\begin{aligned}
\frac{1}{q^{\alpha}-1}+\frac{k-1}{q-1} & =g(\alpha)+(k-1) g(1) \\
& =g\left(\alpha_{1}\right)+\cdots+g\left(\alpha_{k}\right) \\
& >h\left(\alpha_{1}\right)+\cdots+h\left(\alpha_{k}\right) \\
& =\frac{1}{q^{\alpha_{1}}-1}+\cdots+\frac{1}{q^{\alpha_{k}}-1},
\end{aligned}
$$

where we used in the second line that $g$ is linear and that $\alpha+k-1=\alpha_{1}+\cdots+\alpha_{k}$ and in the third line that $g$ is linear and $h$ convex, that $g(\alpha)=h(\alpha)$ and $g(1)=$ $h(1)$ and that $0<\alpha<\alpha_{i}<1$ for every $i \in\{1, \ldots, k\}$.

The final statement in the lemma is easy.

Lemma 3. Let $s_{0}$ be a negative rational number. Let $\gamma, \delta \in R[x]$ be monic polynomials in one variable. Suppose that $\delta$ factors into linear polynomials over $R$ and that all roots of $\delta$ are also roots of $\gamma$. Write

$$
\gamma(x)=\left(\prod_{i \in Q}\left(x-a_{i}\right)^{M_{i}}\right)\left(\prod_{i \in Q^{\prime}} h_{i}^{M_{i}}(x)\right)
$$

where the $a_{i}, i \in Q$, are different elements of $R$, where $h_{i}, i \in Q^{\prime}$, are different irreducible monic polynomials over $R$ of degree at least two, and where $M_{i} \geq 1$ for every $i \in Q \cup Q^{\prime}$. Denote the degree of $h_{i}$ by $d_{i}$. Write also

$$
\delta(x)=\prod_{i \in Q}\left(x-a_{i}\right)^{\mu_{i}-1},
$$

where $\mu_{i} \geq 1$ for every $i \in Q$. Take any $a \in R$ and put $M:=\left(\sum_{i \in Q} M_{i}\right)+$ $\left(\sum_{i \in Q^{\prime}} d_{i} M_{i}\right)$ and $\mu:=\left(\sum_{i \in Q}\left(\mu_{i}-1\right)\right)+1$. Suppose that $0<\alpha:=\mu+s_{0} M$ and $1>\alpha_{i}:=\mu_{i}+s_{0} M_{i}$ for every $i \in Q$ and that $|Q| \geq 2$ or $\left|Q^{\prime}\right| \geq 1$. Then

$$
\left[\int_{R}|\gamma(x)|^{s}|\delta(x)||d x|\right]_{s=s_{0}}^{\mathrm{mc}}<\left[\int_{R}|x-a|^{M s+\mu-1}|d x|\right]_{s=s_{0}}^{\mathrm{mc}} .
$$


Proof. We associate a tree to a monic polynomial $g \in R[x]$ in one variable as follows. If $a_{1}, a_{2} \in R$ are roots of $g$ and if $a_{1} \equiv a_{2} \bmod P^{r}$ and $a_{1} \not \equiv a_{2} \bmod P^{r+1}$, then we associate a vertex to $a_{1}+P^{r}$. If we have an irreducible factor of $g$ of degree at least two, we have associated $r \in \mathbb{N}$ and $b+P^{r}$ to it, and we associate to $b+P^{r}$ a vertex. (If say $a+P^{r}$ occurs several times in this way, we associate to it only one vertex.) We make a tree by connecting vertices in the obvious way, i.e. if we have vertices associated to $a+P^{r}$ and $b+P^{t}$, with $r>t$, then we connect both vertices if $a+P^{r} \subset b+P^{t}$ and for any $c+P^{u}$ corresponding to another vertex we don't have $a+P^{r} \subset c+P^{u} \subset b+P^{t}$. Note that this tree is finite and has one root.

We start at the left hand side of the inequality that we want to prove and we consider the tree associated to $\gamma$. We will construct step by step other integrands for which the associated tree is the previous one except one vertex at the end of the tree. So the tree becomes easier after each step. We will do this until the tree completely disappears. At this stage, the integrand will be the one on the right hand side of the inequality that we want to prove.

We explain the first step. We take a vertex at the end of the tree of $\gamma$. This vertex is associated to an element of $R / P^{r}$ for some $r$, let us say $a_{0}+P^{r}$. Let $a_{1}, \ldots, a_{k}$ be all the roots of $\gamma$ for which $a_{i} \equiv a_{0} \bmod P^{r}$. Note that $a_{i} \not \equiv$ $a_{j} \bmod P^{r+1}$ for $i, j \in\{1, \ldots, k\}$ with $i \neq j$, because we took a vertex at the end of the tree. Let $h_{k+1}, \ldots, h_{l}$ be all the irreducible factors of $\gamma$ to which we have associated $a_{0}+P^{r}$. Write

$$
\gamma(x)=\widetilde{\gamma}(x)\left(x-a_{1}\right)^{M_{1}} \cdots\left(x-a_{k}\right)^{M_{k}} h_{k+1}^{M_{k+1}} \cdots h_{l}^{M_{l}}
$$

and

$$
\delta(x)=\widetilde{\delta}(x)\left(x-a_{1}\right)^{\mu_{1}-1} \cdots\left(x-a_{k}\right)^{\mu_{k}-1} .
$$

Put $M_{0}:=M_{1}+\cdots+M_{k}+d_{k+1} M_{k+1}+\cdots+d_{l} M_{l}$ and $\mu_{0}:=\left(\mu_{1}-1\right)+\cdots+$ $\left(\mu_{k}-1\right)+1$. Put $\gamma_{1}(x)=\widetilde{\gamma}(x)\left(x-a_{0}\right)^{M_{0}}$ and $\delta_{1}(x)=\widetilde{\delta}(x)\left(x-a_{0}\right)^{\mu_{0}-1}$. We have

$$
\begin{aligned}
& {\left[\int_{a_{0}+P^{r}}|\gamma(x)|^{s}|\delta(x)||d x|\right]_{s=s_{0}}^{\mathrm{mc}}} \\
& =\left[\left|\widetilde{\gamma}\left(a_{0}\right)\right|^{s}\left|\widetilde{\delta}\left(a_{0}\right)\right| \int_{a_{0}+P^{r}} \prod_{i=1}^{k}\left|x-a_{i}\right|^{M_{i} s+\mu_{i}-1} \prod_{j=k+1}^{l}\left|h_{j}(x)\right|^{M_{j} s}|d x|\right]_{s=s_{0}}^{\mathrm{mc}} \\
& =\left|\widetilde{\gamma}\left(a_{0}\right)\right|^{s_{0}}\left|\widetilde{\delta}\left(a_{0}\right)\right|\left[\int_{a_{0}+P^{r}} \prod_{i=1}^{k}\left|x-a_{i}\right|^{M_{i} s+\mu_{i}-1} \prod_{j=k+1}^{l}\left|h_{j}(x)\right|^{M_{j} s}|d x|\right]_{s=s_{0}}^{\mathrm{mc}} \\
& <\left|\widetilde{\gamma}\left(a_{0}\right)\right|^{s}\left|\widetilde{\delta}\left(a_{0}\right)\right|\left[\int_{a_{0}+P^{r}}\left|x-a_{0}\right|^{M_{0} s+\mu_{0}-1}|d x|\right]_{s=s_{0}}^{\mathrm{mc}} \\
& =\left[\left|\widetilde{\gamma}\left(a_{0}\right)\right|^{s}\left|\widetilde{\delta}\left(a_{0}\right)\right| \int_{a_{0}+P^{r}}\left|x-a_{0}\right|^{M_{0} s+\mu_{0}-1}|d x|\right]_{s=s_{0}}^{\mathrm{mc}}
\end{aligned}
$$




$$
\begin{aligned}
& =\left[\int_{a_{0}+P^{r}}|\widetilde{\gamma}(x)|^{s}|\widetilde{\delta}(x)|\left|x-a_{0}\right|^{M_{0} s+\mu_{0}-1}|d x|\right]_{s=s_{0}}^{\mathrm{mc}} \\
& =\left[\int_{a_{0}+P^{r}}\left|\gamma_{1}(x)\right|^{s}\left|\delta_{1}(x)\right||d x|\right]_{s=s_{0}}^{\mathrm{mc}},
\end{aligned}
$$

where we used the previous lemma and we used twice that $|\widetilde{\gamma}|$ and $|\widetilde{\delta}|$ are constant on $a_{0}+P^{r}$, and

$$
\begin{aligned}
& {\left[\int_{R \backslash\left(a_{0}+P^{r}\right)}|\gamma(x)|^{s}|\delta(x)||d x|\right]_{s=s_{0}}^{\mathrm{mc}}} \\
& =\left[\int_{R \backslash\left(a_{0}+P^{r}\right)}|\widetilde{\gamma}(x)|^{s}|\widetilde{\delta}(x)| \prod_{i=1}^{k}\left|x-a_{i}\right|^{M_{i} s+\mu_{i}-1} \prod_{j=k+1}^{l}\left|h_{j}(x)\right|^{M_{j} s}|d x|\right]_{s=s_{0}}^{\mathrm{mc}} \\
& =\left[\int_{R \backslash\left(a_{0}+P^{r}\right)}|\widetilde{\gamma}(x)|^{s}|\widetilde{\delta}(x)|\left|x-a_{0}\right|^{M_{0} s+\mu_{0}-1}|d x|\right]_{s=s_{0}}^{\mathrm{mc}} \\
& =\left[\int_{R \backslash\left(a_{0}+P^{r}\right)}\left|\gamma_{1}(x)\right|^{s}\left|\delta_{1}(x)\right||d x|\right]_{s=s_{0}}^{\mathrm{mc}},
\end{aligned}
$$

where we used the last sentence in the formulation of the previous lemma, so that

$$
\left[\int_{R}|\gamma(x)|^{s}|\delta(x)||d x|\right]_{s=s_{0}}^{\mathrm{mc}}<\left[\int_{R}\left|\gamma_{1}(x)\right|^{s}\left|\delta_{1}(x)\right||d x|\right]_{s=s_{0}}^{\mathrm{mc}} .
$$

In the second step, we do the same as in the first step, but use now $\gamma_{1}(x)$ instead of $\gamma(x)$ and $\delta_{1}(x)$ instead of $\delta(x)$. Remark that the $M$ and $\mu$ determined by $\gamma$ and $\delta$ are the same as the analogous ones determined by $\gamma_{1}$ and $\delta_{1}$. Remark also that the tree associated to $\gamma_{1}$ is the tree associated to $\gamma$ with one vertex missing.

Denote the number of vertices of the tree associated to $\gamma$ by $w$. Then, after $w$ steps, the tree completely disappears. If the root of the tree is associated to $a_{0}^{\prime}+P^{r^{\prime}}$, then $\gamma_{w}(x)=\left(x-a_{0}^{\prime}\right)^{M}$ and $\delta_{w}(x)=\left(x-a_{0}^{\prime}\right)^{\mu-1}$. Consequently,

$$
\begin{aligned}
{\left[\int_{R}|\gamma(x)|^{s}|\delta(x)||d x|\right]_{s=s_{0}}^{\mathrm{mc}} } & <\left[\int_{R}\left|\gamma_{1}(x)\right|^{s}\left|\delta_{1}(x)\right||d x|\right]_{s=s_{0}}^{\mathrm{mc}} \\
& <\left[\int_{R}\left|\gamma_{2}(x)\right|^{s}\left|\delta_{2}(x)\right||d x|\right]_{s=s_{0}}^{\mathrm{mc}} \\
& <\cdots\left[\int_{R}\left|\gamma_{w}(x)\right|^{s}\left|\delta_{w}(x)\right||d x|\right]_{s=s_{0}}^{\mathrm{mc}} \\
& <\left[\int_{R}\left|x-a_{0}^{\prime}\right|^{M s+\mu-1}|d x|\right]_{s=s_{0}}^{\mathrm{mc}} \\
& =\left[\int_{R}|x-a|^{M s+\mu-1}|d x|\right]_{s=s_{0}}^{\mathrm{mc}} .
\end{aligned}
$$


In the next proposition, we use the setting explained in the beginning of this section.

Proposition 2. Let $s_{0}:=-\nu_{r} / N_{r}+(2 k \pi \sqrt{-1}) /\left(N_{r} \log q\right)$ be an arbitrary candidate pole of $Z_{f}(s)$ associated to $E_{r}$. Suppose that $\alpha_{i}:=\mu_{i}+s_{0} M_{i}$ is not a multiple of $2 \pi \sqrt{-1} /(\log q)$ for every $i \in S$. Suppose $|S|=3$ and $\left|S^{\prime}\right|=0$. Let $\mathcal{R}$ be the contribution of $E_{r}$ to the residue of $Z_{f}(s)$ at $s_{0}$. Then, $\mathcal{R} \neq 0$.

Proof. Denote the elements of $S$ by 1, 2 and 3. Then the equality (2) says $\alpha_{1}+$ $\alpha_{2}+\alpha_{3}=1+(2 k \pi \sqrt{-1}) /(\log q)$. By applying an affine coordinate transformation, we may assume that

$$
f \circ g_{1} \circ \cdots \circ g_{r-1}=d y_{2}^{M_{1}} y_{1}^{M_{2}}\left(y_{1}-a y_{2}\right)^{M_{3}}+\text { terms of higher degree, }
$$

and

$$
\left(g_{1} \circ \cdots \circ g_{r-1}\right)^{*} d x=\left(e y_{2}^{\mu_{1}-1} y_{1}^{\mu_{2}-1}\left(y_{1}-a y_{2}\right)^{\mu_{3}-1}+\text { terms of higher degree }\right) d y
$$

with $a \in R \backslash P$ and $d, e \in K^{\times}$. We have now that

$$
\begin{aligned}
\mathcal{R} & =|d|^{s_{0}}|e|\left(\frac{q-1}{q} \frac{1}{q^{\alpha_{1}}-1}+\frac{q-1}{q} \frac{1}{q^{\alpha_{2}}-1}+\frac{q-1}{q} \frac{1}{q^{\alpha_{3}}-1}+\frac{q-2}{q}\right) \\
& =|d|^{s_{0}}|e|\left(\frac{1-q^{\alpha_{1}-1}}{1-q^{-\alpha_{1}}} \cdot \frac{1-q^{\alpha_{2}-1}}{1-q^{-\alpha_{2}}} \cdot \frac{1-q^{\alpha_{3}-1}}{1-q^{-\alpha_{3}}}\right) \\
& \neq 0 .
\end{aligned}
$$

The second equality can be checked by a straightforward calculation and is due to Sally and Taibleson [ST].

Remark. (1) The determination of all poles (real and complex) of an absolutely analytically irreducible curve follows now immediately. This was one of the main results of the paper [Ig2] of Igusa. He also used Sally-Taibleson's formula.

(2) However, if $|S| \neq 3$ or $\left|S^{\prime}\right| \neq 0$, it is not clear to us which non-real candidate poles are poles and which are not. It can happen that a real candidate pole is a pole, and that other candidate poles with the same real part are not poles. This is for example the case when $f=x_{1}^{2}+x_{2}^{2}$ and $p=2$ (see [Se1, Example 2.8]).

\section{Poles of Igusa's $p$-adic zeta function}

Let $f \in K\left[x_{1}, x_{2}\right]$ and let $X$ be an open and compact subset of $K^{2}$. Suppose that $f_{\text {red }}$ has only one singular point $P_{0}$ in $X$. Let $g: Y \rightarrow X$ be an embedded resolution of $f$. Write $g=g_{1} \circ \cdots \circ g_{t}: Y=Y_{t} \rightarrow X=Y_{0}$ as a composition of blowing-ups $g_{i}: Y_{i} \rightarrow Y_{i-1}, i \in T_{e}:=\{1, \ldots, t\}$, centered at $P_{i-1} \in Y_{i-1}$. The 
exceptional curve of $g_{i}$ and also the strict transforms of this curve are denoted by $E_{i}$. The closed submanifolds of $Y$ of codimension one which are the zero locus of the strict transform of an irreducible factor of $f$ in $K[x, y]$ are denoted by $E_{j}$, $j \in T_{s}$. The corresponding transforms in $Y_{i}, i \in\{0, \ldots, t-1\}$, are denoted in the same way. Put $T=T_{e} \cup T_{s}$.

In the (dual) embedded resolution graph of the germ of $f$ at $P_{0}$ one associates to each exceptional curve a vertex (represented by a dot) and to each intersection between exceptional curves in $Y$ an edge, connecting the corresponding vertices. We also associate to each analytically irreducible component of the strict transform of the germ of $f$ at $P_{0}$ a vertex (represented by a circle), and to its (unique) intersection with an exceptional curve in $Y$ a corresponding edge. It is clear that this graph is a finite connected tree with all circles end vertices.

Now to each vertex of the embedded resolution graph we associate the corresponding ratio $\nu_{i} / N_{i}$. This makes the embedded resolution graph into an ordered tree. More precisely, the vertices for which the associated number is equal to $\min _{i \in T} \nu_{i} / N_{i}$, together with their edges, form a connected part $\mathcal{M}$ of the embedded resolution graph, and starting from an end vertex of the minimal part $\mathcal{M}$, the numbers $\nu_{i} / N_{i}$ strictly increase along any path in the tree (away from $\mathcal{M}$ ). This follows from relation (2) and the bound on the $\alpha$ 's, which imply for example that there exists at most one $E_{j}$ which intersects a given $E_{r}, r \in T_{e}$, in $Y$ with $\nu_{j} / N_{j}<\nu_{r} / N_{r}$ (see Section 2). For more details, see [Ve2, Theorem 3.3], where the base field is $\mathbb{C}$ instead of $K$, but nevertheless the proof of our statement is similar.

Example. When $f$ is absolutely analytically irreducible at $P_{0}$ with $g$ different Puiseux exponents, then the resolution graph has the form
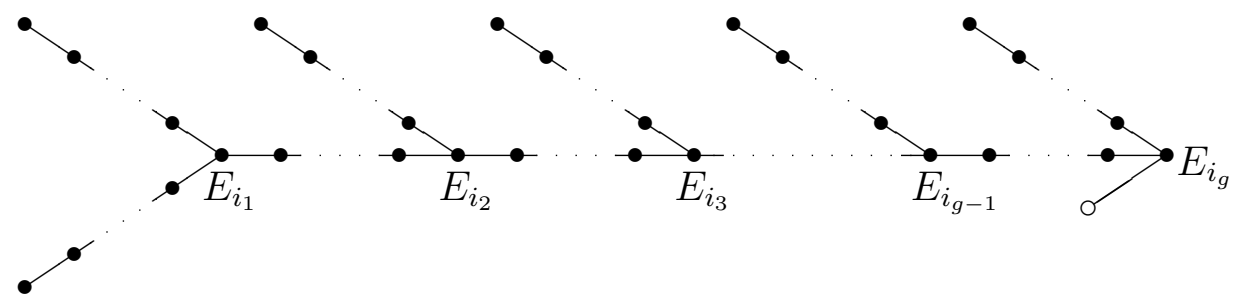

The minimal part $\mathcal{M}$ consists just of $E_{i_{1}}$ (see [St, Corollary 2.1] or [Ve2, Proposition 3.6]).

Theorem. Suppose that we are in the setting of the first paragraph of this section. Then,

1. a real number $s_{0}$ is a pole of order 2 if and only if there exist $i, j \in T$ with $s_{0}=-\nu_{i} / N_{i}=-\nu_{j} / N_{j}$ such that $E_{i}$ and $E_{j}$ intersect on $Y$, moreover, $Z_{f}(s)$ has at most one real pole of order two, and if there is a pole of order two, 
it is the pole closest to the origin,

2. a real number $s_{0} \in\left\{-\nu_{i} / N_{i} \mid i \in T_{e}\right\} \backslash\left\{-\nu_{i} / N_{i} \mid i \in T_{s}\right\}$ which is not a pole of order 2 is a pole of order 1 if and only if there exists at least one $i \in T_{e}$ with $s_{0}=-\nu_{i} / N_{i}$ such that $f \circ g_{1} \circ \cdots \circ g_{i-1}$ is given in local coordinates centered at $P_{i-1}$ by a power series with lowest degree part a homogeneous polynomial which is not a power of a linear polynomial or a product of two such powers, and

3. a real number $s_{0} \in\left\{-\nu_{i} / N_{i} \mid i \in T_{s}\right\}$ which is not a pole of order 2 is a pole of order 1 for a small enough open and compact neighborhood $X$ of $P_{0}$.

Proof. (1) It is clear that there exist $i, j \in T$ with $s_{0}=-\nu_{i} / N_{i}=-\nu_{j} / N_{j}$ such that $E_{i}$ and $E_{j}$ intersect on $Y$ if $s_{0}$ is a real pole of order 2. If $E_{i}$ and $E_{j}$ intersect on $Y$ with $s_{0}=-\nu_{i} / N_{i}=-\nu_{j} / N_{j}$, then the contribution of $P:=E_{i} \cap E_{j}$ to the coefficient $b_{-2}$ in the Laurent series

$$
\frac{b_{-2}}{\left(s-s_{0}\right)^{2}}+\frac{b_{-1}}{s-s_{0}}+b_{0}+b_{1}\left(s-s_{0}\right)+\cdots
$$

of $Z_{f}(s)$ at $s_{0}$ is equal to

$$
|\varepsilon(P)|^{s_{0}}|\eta(P)| \frac{(q-1)^{2}}{q^{2} N_{i} N_{j}(\log q)^{2}}>0,
$$

and consequently, the contributions of different intersecting pairs on $Y$ cannot cancel each other. The other statements follow from the ordered tree structure of the embedded resolution graph.

(2) The 'only if' part is the well known part of Proposition 1 which is due to Loeser. For the other implication, we have to use Proposition 1 and the ordered tree structure of the embedded resolution graph. There are two possibilities. In the first case, $E_{i}$ is the minimal part of the embedded resolution graph. In this case, there is only one contribution to the residue, which is positive. In the other case, there is at least one non-zero contribution to the residue, and all such contributions are negative.

(3) There are two possibilities. In the first case, $s_{0}=-\nu_{i} / N_{i}$, with $i \in T_{s}$ and $E_{i}$ is the minimal part of the embedded resolution graph. In this case, there is only one contribution to the residue, which is positive. In the other case, we take a (small enough) open and compact neighborhood $V$ of $\cup_{i \in T_{e}} E_{i} \subset Y$ on which all the $E_{i}, i \in T_{s}$ with $s_{0}=-\nu_{i} / N_{i}$, have a negative contribution to the residue of $Z_{f}(s)$ at $s_{0}$. The $E_{i}, i \in T_{e}$ with $s_{0}=-\nu_{i} / N_{i}$, have a non-positive contribution to the residue of $Z_{f}(s)$ at $s_{0}$. If we replace $X$ by $g(V)$ or by an open and compact neighborhood of $P$ contained in $g(V)$, we obtain what we want. 
Remark. It follows from the previous theorem and from the result of Loeser mentioned in the introduction that $\operatorname{Re}\left(s_{0}\right)$ is a pole of $Z_{f}(s)$ if $s_{0}$ is a pole of $Z_{f}(s)$. Consequently, we know the set of real parts of poles of $Z_{f}(s)$. This determines the asymptotic behaviour of the number of solutions of the corresponding polynomial congruences (see [Se2] for more information).

\section{References}

[De1] J. Denef, On the degree of Igusa's local zeta function, Amer. J. Math. 109 (1987), 9911008.

[De2] J. Denef, Report on Igusa's local zeta function, Sém. Bourbaki 741, Astérisque 201/202/203 (1991), 359-386.

[DL] J. Denef and F. Loeser, Caractéristique d'Euler-Poincaré, fonctions zêta locales et modifications analytiques, J. Amer. Math. Soc. 5, 4 (1992), 705-720.

[Hi] H. Hironaka, Resolution of singularities of an algebraic variety over a field of characteristic zero, Ann. Math. 79 (1964), 109-326.

[Ig1] J. Igusa, On the first terms of certain asymptotic expansions, Complex Analysis and Algebraic Geometry, Iwanami Shoten (1977), 357-368.

[Ig2] J. Igusa, Complex powers of irreducible algebroid curves, Geometry today, Roma 1984, Progress in Math. 60, Birkhäuser, 1985, pp. 207-230.

[Ig3] J. Igusa, An Introduction to the Theory of Local Zeta Functions, Amer. Math. Soc., Studies in Advanced Mathematics 14, 2000.

[La] R.P. Langlands, Orbital integrals on forms of SL(3), Amer. J. Math. 105 (1983), 465-506.

[Lo] F. Loeser, Fonctions d'Igusa p-adiques et polynômes de Bernstein, Amer. J. Math. 110 (1988), 1-21.

[Me] D. Meuser, On the Poles of a Local Zeta Function for Curves, Invent. Math. 73 (1983), 445-465.

[ST] P.J. Sally and M.H. Taibleson, Special functions on locally compact fields, Acta Math. 116 (1966), 279-309.

[Se1] D. Segers, On the smallest poles of Igusa's p-adic zeta functions, Math. Z. 252 (2006), $429-455$.

[Se2] D. Segers, The asymptotic behaviour of the number of solutions of polynomial congruences, to appear in An. St. Univ. Ovidius Constanta.

[St] L. Strauss, Poles of a two-variable p-adic complex power, Trans. Amer. Math. Soc. 278 (1983), 481-493.

[Ve1] W. Veys, On the poles of Igusa's local zeta function for curves, J. London Math. Soc. 41 (1990), 27-32.

[Ve2] W. Veys, Determination of the poles of the topological zeta function for curves, Manuscripta Math. 87 (1995), 435-448. 
Faculty of Mathematics and Informatics, "Ovidius" University, Constanta, Mamaia Bd. 124, RO 900527 Constanta, Romania

E-mail address: denis@univ-ovidius.ro

University of Leuven, Department of Mathematics, Celestijnenlaan 200B, B3001 LeUven, Belgium

E-mail address: dirk.segers@wis.kuleuven.be

URL: http://wis.kuleuven.be/algebra/segers/segers.htm 